\title{
Evidences of the charge fundamental asymmetry
}

\author{
[The origin of the rotating normal body's magnetic field and \\ the biggest mysteries of the cosmology]
}

\section{Mohsen LUTEPHY}

The faculty of science, Azad University, south Tehran branch, Iran e-mail: lutephy@gmail.com

website: https://www.researchgate.net/profile/Mohsen_Lutephy

\begin{abstract}
There is an infinitesimal small non polarizable electric inertial charge in the individual atoms or in a number neutral matter included to equal number of the electrons and protons. We refer to the pure calculations verified by the experimental laboratorial results, also planetary phenomena and cosmic observations. This inertial electric charge is origin of a part of the measurable magnetic field in addition to self-consistent dynamo, together as complementary origins to generate very complex magnetism of the celestial bodies. Ultimately we refer to the null results of the test experiments for the spinning bodies magnetic field and we see that neither used bodies in the experiments have been number neutral (no equal number of the electrons and protons), nor the simple formula of the Patrick Blackett is accurate in detail. But we have extracted a new formula in agreement for all spinning bodies magnetic fields, in agreement with both terrestrial and cosmic bodies. We verify the effect of electric charge asymmetry in expanding universe and acceleration of the universe expansion and anisotropy of cosmic microwave background and singularity paradox and solar wind and acceleration of solar wind and corona high temperature puzzle as an equilibrium between the gravity and antigravity and mysterious heat generation of the Sun and planets interiors and missing neutrino and the comets plasma tail outward direction and pendulums mysterious precessions and some other mysteries phenomena too.
\end{abstract}

Key words: electric charge asymmetry, celestial magnetic field, Nipher experiment, expanding universe, corona, excess charge, microwave background, solar wind, antigravity, missing neutrino, pendulums mysterious precession, Podkletnov effect

\section{Introduction}

Albert Einstein considered the origin of the Earth's magnetic field, one of the five most important unsolved problems in the physics and generally the 
origin of the celestial bodies magnetic fields is a long time mystery of the science.

It has been developed, the gravity driven magnetic field by relevant scientists to answer to the celestial bodies magnetic fields. Arthur Schuster (Schuster, 1912) initiated such a hypothesis on the base of the gravitational magnetism and Patrick Blackett (Blackett, 1947) formulized it in a simple correlation between magnetic moment and angular momentum of the celestial bodies. Then these theories went to the laboratories to test experimentally.

Blackett simple equation (Blackett, 1947) was showing that his golden rotating cylinder should show a magnetic moment greater than $10^{-9}$ Gauss. But the experiments did not result such an answer (Wilson, 1923) and Patrick Blackett (Blackett, 1952) announced, the failure of these gravitational magnetism theories. After negative result of the Gravitational magnetism theories, the self-consistent dynamo was developing by scientists, similar to the Einstein relativity that after null result of the MichelsonMorely experiments (Michelson and Morely, 1887), the Einstein relativity was growing rapidly.

The second half of the twentieth century, the dynamo theory, describing the process through which a rotating, convecting, and electrically conducting fluid acts to maintain a magnetic field, was used to explain how hot iron in the outer core of the Earth creates a magnetosphere.

Of course there are some controversial evidences opposite with self-consistent Dynamo. For example, a new article by Gregory Ryskin (Ryskin, 2009), is showing that the Earth magnetic field origin should be revisited. In Ryskin's paper the correlation of the secular variation of the geomagnetic field and oceans flows is opposite with the before concept of the core fluids which was theorized reasonable for self-consistent Dynamo.

Thomas Cowling's anti-dynamo theorem (Cowling, 1934) states that no axisymmetric magnetic field can be maintained through a self-sustaining dynamo action by an axially symmetric current and he proved that a dynamo can't exist if both flows and magnetic field are axisymmetric about the same axis. Some more of these anti-dynamo theorems are given in Moffatt (1978) that purely toroidal motion can't produce a dynamo, and neither can purely planar motion. However, a mix of stationary magnetic field and dynamo, strongly can resolve the paradoxes as the wave and particle complementary 
did resolve many paradoxes in the physics.

There are also thermal paradoxes for self-consistent dynamo theory for example wide spread paleomagnetic evidence for a Geodynamo (Tarduno et al., 2010) creates the new core paradox as a puzzle that what powered the Geodynamo prior to inner core nucleation and too as noted by Denis Andrault (Andrault et al., 2016) that:

"the Earth is a thermal engine generating the fundamental processes of geomagnetic field, plate tectonics and volcanism. Large amounts of heat are permanently lost at the surface yielding the classic view of the deep Earth continuously cooling down."

And then we need a source to keep the Earth core temperature almost constant along the billion years and in reality the temperature constancy of the core is needed until the Geodynamo to be stable historically to resolve the thermal paradox.

The magnetic field is correlated with all spinning cosmic bodies especially about the neutron stars. The generation of extreme magnetic field in neutron stars is completely ambiguity in self-consistent dynamo mechanism and finding a dynamo region in so compressed neutron regions in these stars is almost impossible. For example, PSR J1748-246ad is rotating at the surface around the $25 \%$ of the light speed and for such a neutron star, assuming a self-consistent dynamo is almost impossible.

It is clear that we should find a next way for magnetic field generation on the neutron stars. However, it is visible that in the Sun like stars, the dynamo is working well.

It is time now to revisit the theories and never we don't allow to be sure about anything and the history of science is showing that complete sure about the scientific subjects is error. For that after a passing time, some things new are opposing the before ideas we have been completely sure about them and in reality the increase of the paradoxes is the sign for failure of the theories.

It is almost hard to exclude the theory of the self-consistent dynamo and dynamo theory may be actually in agreement with some celestial bodies. But it is too visible that the spinning bodies also have different origin to generate magnetic field.

Here in this paper we return to the origin of the celestial bodies magnetic field and we want to show why the experiments didn't result magnetic field 
for rotating disks in the laboratories.

We have obtained here a universal formula for spinning bodies magnetic field completely in agreement with small and big bodies, whether terrestrial or cosmic and it is all on the base of the electromagnetism not relevant to any type of the gravitational theories, however the formula is very near in shape to the Patrick Blackett simple formula.

We find that the self-consistent dynamo is not the main origin of the planets magnetic field presently whereas that it is yet a compatible theory for some secular variations in the planets magnetic field. But the self-consistent dynamo is yet compatible mainly in some types of the celestial bodies. In reality on the base of the observations we find that both the self-consistent dynamo and stationary magnetic field of the rotating bodies is working in the celestial bodies as the complementary mechanism with different percentages in different bodies.

In addition to the magnetic field interpretations we have resulted here that the electric charge asymmetry is reason of some biggest problems in the cosmology and Earth and planetary sciences. For example, expanding universe and microwave background asymmetry (CMBR) and solar wind and its acceleration and corona puzzle and missing neutrons and comets plasma tail out ward direction and pendulums mysterious precessions and even the inflation theory and singularity paradox and many next mysterious phenomena.

\section{Electric charge asymmetry}

For a very short description of the subject we refer here to some sentences noted by Sujan Sengupta (Sengupta, 2000) that:

"Almost forty years ago Lyttleton and Bondi (1959) suggested that if the electron and proton charge differed by a part in 10 ${ }^{18}$, it could account for the expansion of the Universe. Consequently, interest developed on the issue of the possibility of a tiny charge asymmetry of matter in particular and the Universe in general. The subject gained general acceptance by the scientific community after the experiment of Hillas and Cranshaw (1959). There were several experiments to detect charge asymmetry in matter. However, all these experiments put definite upper limits on the charge asymmetry 
which are much lower than that required by Lyttleton and Bondi (1959). It is worth mentioning that the strength of the electromagnetic interaction is $10^{39}$ times stronger than the gravitational interaction and so any tiny electric charge asymmetry could have profound consequence. Considering the anisotropy in the cosmic microwave background radiation (CMBR). Sengupta and Pal (1996) found that the present excess number density of particles with charge $e$ over the particles of opposite and equal charge must be less than $8.5 \times 10^{26} \mathrm{~cm}^{-3}$."

And as noted at (Sengupta and Pal, 1996) that:

"We point out that an overall electric charge asymmetry in the universe will generate an anisotropy in the cosmic microwave background radiation $(C M B R)$ via the Sach-Wolfe effect. From this, an upper limit on the electric charge asymmetry over a cosmological scale is found using the COBE data, which implies $\triangle T T \leq 10^{-4}$ for the $C M B R$. The same argument constrains the charge of degenerate neutrinos."

But we refer to a very precise experiment in Cavendish type series which enabling to report experimentally a repulsive force in comparison with attractive gravity. Francis E. Nipher in several consequent experiments (Nipher, 1916, 1917, 1918, 1920) applied electric induction into the metallic bodies as noted at New York Times (19 September 1917) that:

"If electricity can alter the gravitational attraction of the bodies used in my experiments," he said, "the same force can alter the Earth's attraction. If the negative electricity could be drawn from the Earth's surface, gravitational attraction suddenly would cease and the cohesion of the Earth's surface would be disastrously affected."

In a pamphlet issued (November 8, 1917), Prof. Nipher supplies experimental evidence that gravitational attraction can not only be suspended or nullified by the electrical current, but it actually can be transformed into "gravitational repulsion"! All during the summer of 1917, Prof. Nipher had his apparatus in almost continuous operation, and the experiments have been repeated time and again, always with the same result.

Before connecting any form of electric current to the modified Cavendish apparatus, Prof. Nipher took special precaution to carefully screen the moving element from any electrostatic or electromagnetic effects. His apparatus briefly consists of two large lead spheres ten inches in diameter, resting upon heavy sheets of hard rubber. Two small lead balls, each one inch in 
diameter, were now suspended from two silk threads, stationed at the sides of the two large lead spheres, from which they were separated by a little distance. Moreover, the suspended balls were insulated elaborately from the large spheres by enclosing them first airtight in a long wooden box, which was also covered with tinned iron sheets as well as cardboard sheets and there was, furthermore, a metal shield between the box and the large metal spheres.

In his first experiments, Prof. Nipher applied a high tension current from a static machine to the large lead balls (Fig. 1).

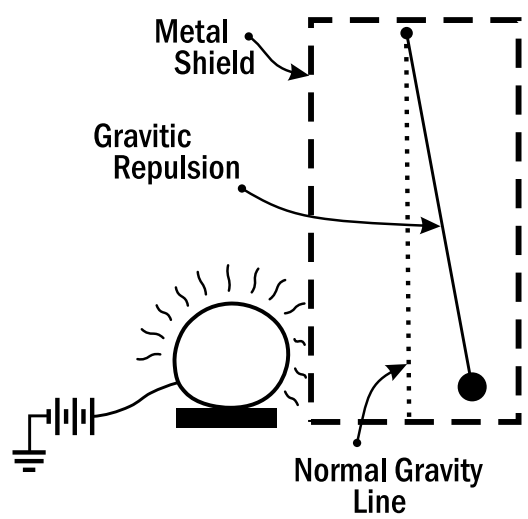

Fig. 1. Gravitational repulsion caused between large \& small masses. Current on.

In one of these experiments the masses were "repelled" by a force nearly twice as great as the initial gravitational repulsion.

First, a direct current of 20 Amperes as sent through the two large masses, but no effect on the suspended masses could be detected. Next, an alternating current of 20 Amperes was sent through the two masses, with the result that the gravitational attraction was quickly reduced to zero, and not only that but in 15 to 20 minutes the small lead spheres had moved over one-half as much to the opposite direction as the distance they had been attracted originally towards the large masses. Thus gravitation had not only been completely nullified, but it was actually reversed.

In further experiments Prof. Nipher decided to check his results. To do this he replaced the large solid lead spheres with two metal boxes, each filled with loose cotton batting. These hollow boxes (having practically no mass) 
rested upon insulators. The metal boxes were then charged in every way that the solid lead spheres had been, but not the slightest change in the position of the lead balls could be detected. This would seem to prove conclusively that the "repulsion" and "gravitational nullification" effects that he had produced when the solid balls were electrically charged were genuine and based undoubtedly on a true inter-atomic electrical reaction, and not upon any form of electrostatic or electromagnetic effects between the large and small masses.

If we consider simply the atoms to be electrified inertial as an electric charge asymmetry for electron and proton, it is clear that a mass included to the equal number of the electrons and protons will have an infinitesimal small inertial electric charge for infinitesimal small difference between the electric charge of the electron and proton. But on the base of the electric equilibrium, clearly along the time, the matter will be transferred to the electrical neutral body by absorption of the excess electrons involved in the matter between other particles (Fig. 2).

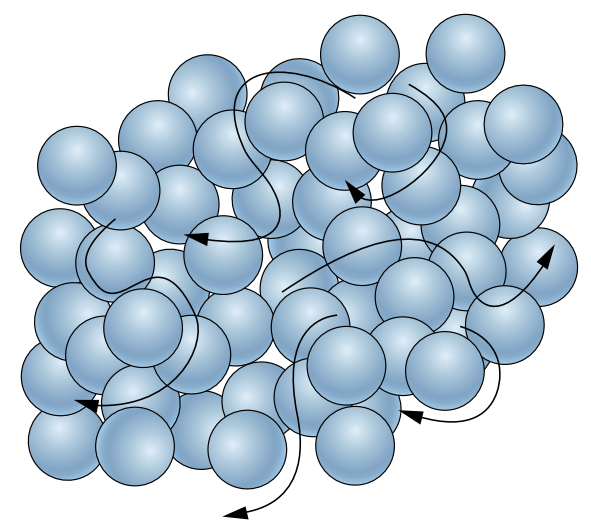

Fig. 2. Involving excess electrons in the matter.

This is a next magic of the quantum mechanics which even violates the Dirichlet principle or pigeonhole principle. Because that here we have $N_{p}$ number of the protons and $N_{e}$ number of the electrons and ultimately $N_{e}$ number of the electrons are situated in the $N_{p}$ number of the protons with $N_{p}<N_{e}$. So that ultimately we have an electrically neutral matter with different number of the electrons and protons and all electrons will be similar. However, the atoms individually are electrical, but additionally we have 
electrically neutral matter and this is reason, why the rotation of the usual disks doesn't result the magnetic field whereas that fundamentally a matter included to the equal number of the electrons and protons should result the magnetic field. Then it is clear that the electrodynamics should be reformed and this modification has been realized by some scientists before, for example as noted at (Lyttleton and Bondi, 1959) that:

"If creation of matter, and also necessarily charge, is assumed, the Maxwell equations must be modified to avoid strict conservation. The appropriate modification is shown to involve additional terms in the current and chargedensity equations proportional to the vector and scalar potentials."

Weber electrodynamics (Assis and Silva, 2000) is a modified electrodynamics and there are also some theories to modify electrodynamics. But for a natural modification of the electrodynamics on the base of the Mach's inertia principle or generally Mach's mechanics (Mach, 1872, 1883, 1911, 1960), we may refer to the book "MOED: the modification of electrodynamics by Mach's inertia principle" (Lutephy, 2016). MOED (Modification of Electro Dynamics) modifies electrodynamics by Mach's mechanics as the universal interconnection of the electric forces so that the Coulomb force is generated in fully relational dynamics (mere ordering upon actual objects) and the electric charge is depended to the large scale electric charge distribution and such a change in the electric charge of the fundamental particles by dependency to the universal electric potential energy is in reality a result of the Mach's mechanics as noted by Sachs and Roy (2003) that:

"We have seen that the qualities of localized matter, such as the inertial mass or electric charge of 'elementary particles', are really only measures of their interactions within a closed system of matter, between these entities and the rest of the system. Thus their values are dependent, numerically, on the rest of the matter of the closed system, of which they are elementary, inseparable constituents. Their masses and electric charges are then measures of coupling within a closed system, not intrinsic properties of 'things' of matter."

By the way when we have a body included to equal number of the electrons and protons, this body has inertial electric charge and to visit this inertial charged body in the laboratory we need to exclude the excess electrons by physical ways. For example, it was used AC voltage into the metallic bodies by Francis E. Nipher in his experiments and it was observed an 
electric repulsive force between metallic bodies. By AC voltage, the excess electrons are assumed to be freed from atomic links transmitted to the surface of the metallic ball to appear the electric repulsive force between these bodies, where the Faraday cage was shielding in the Nipher apparatus, the electric effect of the freed electrons distributed on the surface whereas that Faraday cage not enabling to shield the effect of the non-polarizable inertial electric charge.

This non polarizable inertial charge is not affecting on the polarizable charge means not affecting on the individual free electric charges. This character is the most important feature of the inertial non polarizable electric charges, reasoning to appear mysterious phenomena in the solar system and the cosmos. Clearly this inertial non polarizable electric charge is not possible to be shielded by Faraday cage whereas that Faraday cage shields the polarizable electric charge. Of course it is possible that non-interaction of the atomic level electric charge with free electric charges to be dependent also to the quantum effect of the scale. Because that the inertial electric charge is very tiny electric charge, but free charges are very larger and then probably such a huge difference in the scale of these electric charges reasoning to avoid from interaction of them and such a probable scale dependency of the interaction can be treated by scientists in the future.

Referring to the Nipher experiments, results that this repulsive force between metallic spheres in actual size is at least two times greater than the attractive gravity as mentioned above and then we have for a pair of linked $e-p$ (electron-proton) that:

$k \frac{\left(q_{e-p}^{\&}\right)^{2}}{r^{2}}=2 \frac{G m_{e-p}^{2}}{r^{2}}$,

$q_{e-p}^{\&}=\sqrt{\frac{2 G}{k} m_{e-p}}$,

where $q_{e-p}^{\&}$ is inertial electric charge of a pair of linked electron-proton and $m_{e-p}$ is the mass of each pair.

Then a pair of linked electron-proton have an inertial electric charge at least equal to:

$q_{e-p}^{\&} \cong 2 \times 10^{-37} C$. 
This inertial electric charge is very smaller than the electric charge of an electron. But as we see it is possible to detect by precise measurements in the laboratory.

By the way on the base of the electric charge asymmetry calculated by Nipher experiments analysis, the additional force between two normal bodies (equal number of electrons and protons) $m$ and $m^{\prime}$ is addition of the inertial electric force and pure gravity. Then in actual sizes based on the Nipher experiments for two normal masses $m$ and $m^{\prime}$ in a distance $R$, we have an additional repulsive force $F$ that:

$F=-G \frac{m m^{\prime}}{R^{2}}$.

Now consider schematically a spherical universe with constant density. The acceleration of the universe on a point in radii $r$ because of shell theorem is:

$a_{r}=\frac{\mathrm{d} v}{\mathrm{~d} t} \rightarrow-\frac{G \int_{0}^{r} \mathrm{~d} m}{r^{2}} d r=v \mathrm{~d} v$,

and because that $\int_{0}^{r} \mathrm{~d} m=\rho_{U} V_{r}=\rho_{U} \frac{4}{3} \pi r^{3}$ and substituting this equation in the Eq. (5) implies:

$-\frac{4}{3} \pi G \rho_{U} r \mathrm{~d} r=v \mathrm{~d} v$.

Universe is expanding from an initial small radii and then by integrating from zero it is resulted by Nipher experiments that:

$v=\frac{1}{\sqrt{2}} H r \mid H=\sqrt{\frac{8}{3} \pi G \rho_{U}}$.

To obtain the Hubble law $v=H r$ by the cosmic bodies charge asymmetry we need to reform the Eq. (2) as:

$q_{e-p}^{\&}=\sqrt{\frac{3 G}{k} m_{e-p}}$,

and then the amplitude of the charge asymmetry calculated by Hubble expansion is: 
$q_{e-p}^{\&}=2.44 \times 10^{-37} \mathrm{C}$.

Then referring to the Hubble constant is showing that the electric charge asymmetry obtained by universe expansion should be 1.22 times larger than that of measured by Nipher experiments as a reason to understand that the Nipher balls have not been completely ideal included to the perfect equal number of electrons and protons and this is natural because that the actual size is ever lesser than the ideal and we understand that the normal part of Nipher metallic spheres has been $80 \%$ that of the cosmic matter.

Nipher type experiments is a way to reveal the numerically neutral bodies with equal number of electrons and protons. But, there are other ways too. For example, using heat to exclude excess electrons from their linked level and there are treats in the cosmic and planetary phenomenology too. In 1993, the Nobel Prize in Physics was awarded to Russell Hulse and Joseph Taylor of Princeton University for their 1974 discovery of a pulsar, designated PSR1913+16, in a binary system, in orbit with another star around a common centre of mass. This binary pulsar was under study by Sujan Sengupta (Sengupta, 2000) as an example to reveal the electron-proton charge asymmetry. As noted by Sengupta (2000) that:

"A binary stellar system loses its orbital rotational energy by the emission of gravity wave. If there exists a charge asymmetry between electron and proton, then the system would also lose its orbital rotational energy due to electric dipole radiation provided the masses of the two objects are not exactly equal."

And too that:

"Considering this fact and adopting the observed data of the binary pulsar PSR B1913+16 an upper bound on the electric charge asymmetry between electron and proton is obtained which is more stringent than the existing bounds."

Referring to the calculations of the Sujan Sengupta it is visible a bound amplitude for charge asymmetry as

$\left|q_{e-p}^{\&}\right| \leq 3.2 \times 10^{-20} e$.

This amplitude is almost $2 \%$ that of calculated at Eq. (9). But these different amplitudes are not opposing each other because that a complete number neutral body that $N_{e}=N_{P}$, is an ideal body and actually the celestial bodies are not completely normal. It is not hard to conceive that the 
stars are neutralized by electric equilibrium and the Sun strongly is now not a perfect number neutral body suppose main of the Sun's mass has been neutralized electrically up to now. But the percentage of the neutralization is different in different stars. Interestingly we will see that the magnetic field of the Sun is showing the same about $2 \%$ normal body visible for PSR B1913+16.

The absorption of the excess electrons is a mechanism that it is reason to separate the star's interior to the two different main parts which a part is electrically neutralized part and the next part is numerically neutral or normal. How much the age of the star to be rather, its electric neutralization is rather too. About the Sun the evidences are showing that the normal part exists at the convection zone. The absorption of the electrons by normal matter will transfer it to a pure gravity shape.

About the PSR B1913+16 also it was resulted that just $2 \%$ that of this system is normal as a source of electric dipole radiation responsible to lose the orbital energy and this is in agreement with observations for neutron stars' magnetic field which with almost constant mass and constant spinning rate, the neutron stars have widely different amplitudes of the magnetic fields. In reality, neutrons in normal body are under the electric repulsive force and then stability of the neutrons in the neutron stars needs intermediate exchange particles for example electrons, similar to the system of atoms nucleons. Then neutron stars too are being neutralized electrically by exchange particles and it is mistake to consider all the neutron stars as a completely normal body.

The heat generation in the planets has been a long time mystery and newly Andrault et al. (2016) claiming that lunar tidal effect is responsible to keep the temperature almost in a constant size in the planets interior whereas that such a mechanism is so far to accept. It is clear that the planets interior should be cooled along the billion years whereas that experiments are showing that temperature has been almost constant and this is a paradox.

The force between atoms is addition of the gravity and antigravity in a normal body. When this normal body is neutralized electrically then just remains gravity. By Eq. (8) the antigravity is three times stronger than the gravity and then in electrically neutralization of a normal body we have a heat generation as great as two times of the total gravitational potential 
energy of the body. Now if we consider a normal sphere with mass $M$ and radius $R$, then by definition of the total potential energy of this sphere we have a total heat generation as

$H=2 G \frac{M^{2}}{R}$.

If we consider the Earth initially as a normal body transferring gradually to the electrically neutral body among the time, then total heat generation of the Earth by Eq. (11) is $10^{33} \mathrm{~J}$ and this is in agreement with billion years heat generation of the Earth in average size of $4 \times 10^{15} \mathrm{~W}$. In the planets the mechanism of this phenomenon is that the absorption of the excess electrons causes ever to create heat at the bottom of the outer core which it is a normal shell. This mechanism is reason to increase ever the inner core boundary by addition of the outer core to inner core because of absorption of the excess electrons at the bottom of the outer core. This heat generation will be continued until that all the outer core be transferred to the shape of the inner core and probably this is what it has been happened for Mars which has tiny abnormal magnetic field. Inner core is electrically neutralized by excess electrons and then the additional inter-atomic force is just gravity in the inner core whereas that outer core inter-atomic force is antigravity two times as great as the gravity and this phenomenon causes to condensate the inner core highly in comparison with the outer core and probably inner core solidity.

Scientifically measurements and calculation by Raymond Davis (Davis, 1964) and John Bahcall (Bahcall, 1964) on the solar heat generation by fusion reaction was showing that the generated heat is $1 / 3$ rather we wait from the fusion reaction in the Sun. The assumed energy of the fusion reaction is $1 / 3$ rather than we observe from accounting the neutrinos. The discrepancy between the number of predicted neutrinos and the number Raymond Davis measured became known as "The Solar Neutrino Problem" or in new popular contexts "The Mystery of the Missing Neutrinos".

But here we see that there is no missing neutrino and in addition to the fusion reaction we have a permanent generation of the heat by transformation of the repulsive force of the atoms to the gravitational force.

Calculations on the mass of the Sun's shells is showing agreement between anti gravitational heat generation in the Sun and heat needed to resolve the missing neutrino puzzle. Absorption of the cosmic electrons at 
the Sun or rejection of the positive charges is reason to generate heat by transformation of antigravity to the gravity in the convection zone. In reality the convection zone is divided to the two shells similar to the planets core. A shell is inner convection zone included to the electrically neutral matter by linked excess electrons and an outer convection zone included to the normal matter with equal number of the electrons and protons. Then ever there is a heat generation at the boundary between the inner convection zone and outer convection zone and gradually the outer convection zone is transferred to the inner convection zone similar to the transformation of the outer core to the inner core in the terrestrial planets. The heat generation by electro antigravity at the bottom of the outer convection zone causes to appear convectional cells over the outer convection zone as a source for granulation in the Sun's surface. Also this convection in the outer convection zone as a rotating shell is reasonable to create self-consistent dynamo.

But in the Sun's radiation zone boundary we have a next heat generation by transformation of the antigravity to the gravity. We refer to the reality that the electrically neutralized matter by excess electrons are divided to the two shapes. When we have a usual matter electrically neutralized by excess electrons then all electrons are similar involving between protons with no difference. Then all electrons are linked to the protons and then excess electrons are non-polarizable. But when the temperature is rising highly larger than a critical temperature, the excess electrons become polarizable and this means that matter want to stay normal with equal number of linked electrons and protons in high temperature. Then if we use from two high temperature disks in an experiment (rather than critical temperature) and shielding by a Faraday cage then against the usual matter we will observe antigravity instead gravity similar to what it was visited at the Nipher experiments. These unlinked excess electrons in high temperature matter neutralizes electrically the matter in the absence of shielding effect just in large scale. But we should notice that strongly the nearby links are yet affecting together by antigravity for that just in this condition, the excess electrons are polarizable against the linked excess electrons.

Then for each matter there is a critical temperature as a transition point between linked excess electrons and unlinked excess electrons. It is now soon to discuss this topic accurately and it needs to be continued by scientists in detail. 
Unlinked excess electrons are appeared when the temperature is higher than the critical temperature. But when the temperature comes down then unlinked excess electrons are transferred to the linked level and generating heat.

In reality the Sun radiation zone is included to the hot plasma with temperature rather than the critical temperature included to the unlinked excess electrons whereas that inner convection zone is a low temperate plasma included to the linked excess electrons and normal plasma exist in the outer convection zone. This is reason that the temperature at the radiation zone boundary to be ever constant for that by cooling plasma at the boundary of the radiation zone along the time, unlinked excess electrons are transferred to the linked level in the bottom of the convection zone and then generation of heat at the boundary of the radiation zone avoids from decrease of the temperature at this boundary as a reason for constancy of the temperature at the boundary of radiation zone and as a reason to control the Sun's heat at a constant rate naturally as a natural thermostat. It is manifest that constancy of the Sun radiation is important for life in the Earth.

The heat generation at the radiation zone boundary as a kinematic energy can also generate convective overshoot as a phenomenon of convection carrying material beyond an unstable region of tachocline (Gilman, 2000) into a stratified stable region over a thin shell between radiation zone and convection zone. This thin shell between radiation zone and convection zone is the same what scientists have named it overshooting zone which computers are showing a value of magnetic field for that (Fig. 3).

We may consider also a high temperature plasma disk covered by metallic screens, rotating in high speed to observe magnetic field generated by inertial electric charge of the hot plasma as a new discovery.

By the way, we see that the Sun like stars are not completely normal suppose mainly transferred to the electrically neutral matter by absorption of the excess electrons or excluding the positive nucleons. This mechanism is directly resulting the source for cosmic rays. The electrically neutralization of the cosmos is accompanied with generation of the cosmic rays as a source of cosmic sea of the ionized particles, reasoning to generate shielding effect for unlinked excess electrons in high temperature plasma in large scale systems like the stars' interior.

In reality the absorption of the excess electrons and rejection of the pos- 


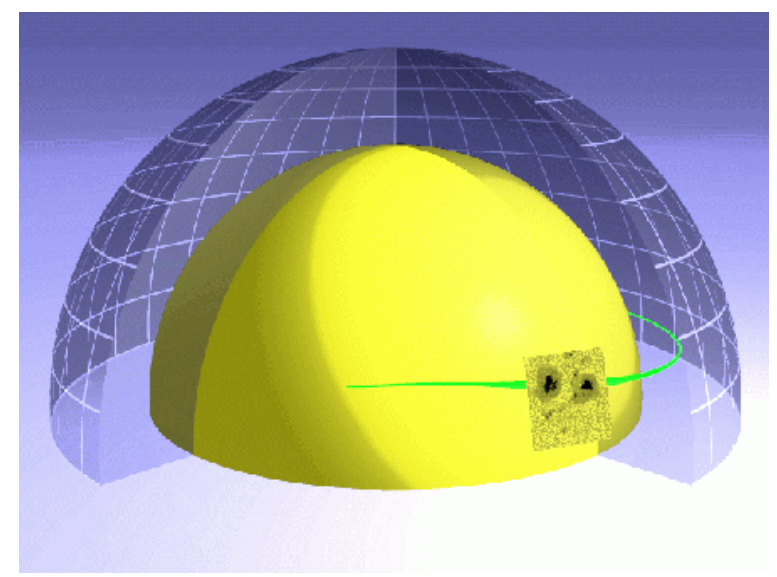

Fig. 3. Credit image: Peter Calligari, Fernando Moreno-Insertis and Manfred Schüssler. (The yellow hemisphere represents the bottom of the convection zone and the transparent one represents its surface. The green line is a bundle of magnetic field lines which has risen from the bottom of the convection zone up to the surface. The little superimposed picture with the two sunspots shows how the bundle of magnetic field lines appears to us when it emerges at the surface.)

itive ions are accompanied and then the total number of the positive cosmic rays is equal to the number of the excess electrons absorbed by matter in the stars and celestial bodies. We can say that concentration of the excess electrons in the stars is accompanied with distribution of positive nucleons in the cosmos and then all the stars and planets and galaxies are floating in a universal sea of the positive cosmic rays.

Generally, we have several clear items that:

a) In the universe the number of the electrons and protons is equal.

b) The number of electrons and protons is conserved if there is no creation of matter and disappearing of the matter.

c) The density of excess electrons is proportional with mean density of the baryonic matter.

d) The density of the cosmic ray is more where the point is farer from the massive areas (Voids).

And then it is appeared a formula for constancy of addition of the excess electrons density and cosmic ray density as: 
$\rho_{\text {excess }}+\rho_{\text {sea }}=k$.

By this formula we find that the vacuum distance between the galaxies is the space with more density of the cosmic ray sea and this means that the large scale vacuum between the galaxies cause to shield highly polarizable electric charges. on the other hand, referring to the Poisson equation of screening effect as the dielectric generated by fluid medium (Kamrin and Koval, 2012), the distance between the galaxies is a factor to increase the screening effect and then the shielding effect is increasing between galaxies. Ultimately we understand that there is a transition radius which in that radii, the gravity is transferred to the antigravity by increase of the shielding effect for unlinked excess electrons in the stars as a reason for gravity at galaxy scale and antigravity at inter galactic scales and larger scales responsible for universe expansion.

\section{The origin of the rotating normal body's magnetic field and cosmic big mysteries}

There are several theories about non-electrical spinning bodies magnetism initiated by Arthur Schuster (Schuster, 1912). On the same process, Patrick Blackett (Blackett, 1947) on the planetary observations did result a phenomenological formula for magnetic moment of non-electrical rotating bodies. His formula went to test in the laboratory. But the experiments were not success to show the predicted magnetic field. For example, Wilson (1923) reported null result and Blackett (1947) experiment didn't show any result for rotating gold cylinder, however the accuracy of the devices was adequate. But here we refer to a reality that each body, whether terrestrial or cosmic is divided to the two parts:

a) Electrically neutral part included to the excess electrons.

b) Number neutral or normal part included to the equal number of the electrons and protons.

Fundamental charge asymmetry is reason that each normal spinning body does generate a magnetic field. For a spherical spinning normal body by assuming a charge density $\rho_{e}$, from definition of the magnetic field at poles and surface we have: 
$B=\frac{8 \pi k}{3 c^{2}} \frac{1}{R^{3}} \omega \int_{0}^{R} \rho_{e} r^{4} \mathrm{~d} r$.

So that $k$ is electric constant and $c$ is light speed and $\omega$ is angular velocity and $R$ is radius and $r$ is distance from the center.

By Eq. (8), the charge density is transferred to the mass density as:

$\rho_{e}=\rho \sqrt{\frac{3 G}{k}}$.

Substituting this equation in the Eq. (13) results a magnetic field at poles of a spherical normal rotating body that:

$B=\frac{8 \pi}{3 c^{2}} \sqrt{3 G k} \frac{\omega}{R^{3}} \int_{0}^{R} \rho r^{4} \mathrm{~d} r$.

Then mathematically on the definition of angular momentum $L$ and the moment of inertia $I$ we obtain by Eq. (13) that:

$B R^{3}=\frac{2}{3 c^{2}} \sqrt{3 G k} \omega I$

$B R^{3}=\frac{2}{3 c^{2}} \sqrt{3 G k} L$

By relation of the magnetic field $B$ to the magnetic moment $p$ that $4 \pi B R^{3}=2 \mu_{0} p$ we obtain by Eq. (17) that:

$p=\frac{\sqrt{G}}{\sqrt{3} \sqrt{k}}$.

This is similar to the Patrick Blackett formula, both in shape and size.

This equation for magnetic moment of rotating normal body is adequate to show magnetic moment in a precise experiment similar to the Blackett experiments which it was adequate to show a magnetic field larger than $10^{-13} \mathrm{~T}$.

But the measurements were not success to show magnetic field for example null result reported by Blackett (1952) and too reported null result by Wilson (1923). But here in this paper we see that there is a big error in 
the measurements about the using rotating bodies. The bodies have been electrically neutral and electrically neutral body in their theories of Gravitational magnetism should result magnetic field whereas that gravitational magnetism doesn't work right suppose magnetic field is ultimately possible to be generated by electric charge torques and the electric charge asymmetry reasons the magnetic field from rotation of normal bodies included to equal number of electrons and protons.

Simple bodies in the Earth surface are all electrically neutralized by excess electrons and they are not normal and then using these bodies in the experiments to detect magnetic field by their spinning will not generate magnetic field.

Initially it needs to exclude the excess electrons and this rejection or shielding requires some especial techniques and possibly the scientists in the future will discover several ways. But referring to the Nipher experiments is showing that applying AC voltage in the same manner the Nipher used, is suitable for excess electrons excluding. Rotating disk should be under a suitable level of $\mathrm{AC}$ voltage and such a rotating disk can show a magnetic field in the experiments when excited excess electrons are shielded. Strongly by such a rotating metallic disk in Nipher type experiments, we will observe a value of magnetic field as a verification for charge fundamental asymmetry.

Similar measurement has been applied before by Eugene Podkletnov (Podkletnov and Nieminen, 1992; Podkletnov and Vuorinen, 1996; Podkletnov, 1997). Eugene Podkletnov did use a super conducting rotating disk under high frequency AC voltage and it was visited a few percent loss of the weight for a body hanging above the disk. Podkletnov effect is now a mystery in the science. But we see here a similarity between Nipher outstanding sphere which was under AC voltage and Podkletnov rotating superconducting disk under $\mathrm{AC}$ voltage.

As it is visible in the Nipher experiments, the metallic sphere under the $\mathrm{AC}$ voltage is reason to induct electromagnetic effect to an electrically neutral metallic body inside the Faraday cage which it causes to transfer it to the side of a normal body. Such an electromagnetic induction is being observed between conductive cores of the planets when they are under Sun's magnetic field variation (Lutephy, 2018) reasoning external driven planetary origin of the geomagnetic jerks and LOD variations and relevant gravity 
anomalies. Here in the Podkletnov effect we see that he has used from a superconducting rotating disk, inducting electromagnetically on the test mass above the disk, reasoning to transfer it to the side of a normal body including a percentage of non-polarizable inertial electric charge. Then it has been generated an electric inertial force between the Earth's outer core inertial charge and test mass used by Eugene Podkletnov, above the rotating superconducting disk which the total effect is reduced highly by the Earth's mantle. Of course it should be noted that dielectric effect of the Earth's mantle is working to decrease electro anti gravitational effect of the Earth's interior inertial electric charge.

The electric force between the Earth outer core and Podkletnov experiment test mass is possible to calculate purely by electric charge asymmetry. If we assume an ideal shape of the Podkletnov test mass as the perfect normal body and we render from dielectric effect of the mantle, then from the Eq. (9) we have an acceleration between the Earth's outer core and Podkletnov ideal test mass generated by Coulomb force that:

$a=k \frac{M_{\text {outer-core }} \sqrt{\frac{3 G}{k}} m_{\text {disk }} \sqrt{\frac{3 G}{k}}}{\left(\sim R_{\text {earth }}\right)^{2} m_{\text {disk }}}=-9.8\left(\mathrm{~m} / \mathrm{s}^{2}\right)$.

And this equation results an electro antigravity on the test mass exactly inverse of gravity as a complete buoyancy.

But we need to notice that the Earth's mantle is a very big dielectric which it causes to decrease highly the effect of the Earth's antigravity in the near Earth's surface on the normal bodies and also it is not clear how much Podkletnov measurement has been success to exclude excess electrons from the test mass transferring them to unlinked level. In fact, the equation (19) is showing that if the excess electrons to be completely excluded then in the absence of the earth big mantle dielectric we can prevail to the gravity and it seems this is what we observe in some versions of the flying saucers and we see that the ultimate power of the Podkletnov machine is perfect buoyancy of bodies at Earth surface.

Then in the Nipher type experiments if we use a method to measure the pendulums weight, when we apply $\mathrm{AC}$ voltage, it should be visited the loss of pendulums weight as a prediction of the electric charge asymmetry. However, the Earth's mantle decreases the effect highly, but when we have observed a weight loss in the Podkletnov experiment we should see it in this 
assumed experiment too.

Nipher did not use any weight measurement and then for such a prediction in Cavendish type measurements it needs to repeat the measurement carefully again included to the weight measurements similar to measurements by electrically charged torsion pendulums used by Erwin Saxl (Saxl, 1964).

In reality the measurement of this buoyancy at the Earth surface is hard because of the Earth's mantle dielectric effect and it needs some measurements in high latitudes and in reality for direct effect of the Sun and planets on the test masses in the Earth surface because of the earth inertial system we need to exist from the Earth inertial boundary otherwise we will detect null result exactly similar to the null result of the Michelson-Morely experiments (Michelson and Morely, 1887). This subject is itself a different issue on specification of the Geodynamo we can continue it in the book "MOED" (Lutephy, 2016) which we have resolved logically the puzzles of the flat earth theory.

We can use from pendulums to measure the electro anti-gravitational effect of the Moon and Sun and planets on the Earth which can generate drag forces on these pendulums because of differential effect of the anti-gravity as a kind of tidal force. Then drag forces generate relevant precessions and variations in oscillation of the pendulums. Such a variation by electro anti-gravity in the pendulums precessions and periods is appeared in the eclipse times or syzygy effects where we have noticeable changes in the Earth electromagnetic features detected initially by Maurice Allais (Allais, $1959 a, 1959 b)$ as noted by Maurice Allais that:

"The observed effects are only seen when the pendulum is moving. They are not connected with the intensity of weight (gravimetry), but with the variation of weight in the space swept by the pendulum. Actually, while the movement of the plane of oscillation of the pendulum is inexplicable by the theory of gravitation, the deviations from the vertical are explained perfectly by that theory. The deviations from the vertical ... correspond to a static phenomenon, while $m$ experiments correspond to a dynamic phenomenon."

Other scientists have tried in this way and yet the pendulum measurements are being continued. For example, Romanian physicists Jeverdan et al. $(1961,1981)$ did observe the Allais effect and the so-called JeverdanRusu-Antonescu effect or Jeverdan effect as the change of oscillation period 
of a pendulum during an eclipse. Also a result during the annular solar eclipse of September 22, 2006 has been presented by a Romanian team, with a quantization of the behavior of the paraconical pendulum (Popescu and Olenici, 2007).

Various other experiments using atomic clocks and gravimeters instead the pendulums also recorded significant anomalous gravitational effects (Zhou et al., 1995; Mishra et al., 1997; Wang et al., 2000). But gravimeters never detected any report comparable in size with the pendulums mysterious effect as a paradox about the incompatibility between the pendulums and gravimeters measurements. The direction of the pendulums effects is aligned with gravitational tidal forces but amplitudes are not in agreement with gravitational theories. But electro antigravity is not in proportion with total mass of the Sun and planets and Moon versus the gravity theories suppose dependent on the normal part of these celestial bodies and also dependent to the electrical screening effects. These effects of the Moon and Sun have been reported by Maurice Allais (Allais, 1999) as monthly and seasonal changes in the precession of the pendulums. Sun and Moon have electro anti-gravitational force on the Earth and then it will be appeared tidal effects on the pendulums and the tidal forces as the drag forces based on the Pippard precession (Pippard, 1988) generate such a phenomenon so that referring to the Pippard solution (Pippard, 1988) for spherically oscillating pendulum on the perturbations it is deduced that:

$\Omega_{p}=\frac{a_{a z}}{2 A \omega} \cos \left(2\left(\theta-\Sigma_{i}\right)\right)$.

So that $\Sigma$ is direction of the force on the azimuth plane and $\theta$ is direction of major axis $A$ with angular velocity $\omega$ and $a_{a z}$ is component of acceleration on azimuth plane.

This equation results in each moment a precession for pendulums in relation with direction of the oscillation plane and direction of the drag force. By Pippard solution we observe that discrete forces occurring about the periods larger than a half of oscillation of the pendulums will generate angular velocity, no requirement to continuum force. Then discrete variations in the Normalization of the Earth by electromagnetic influences will cause to appear continuum precessions whereas that gravimeters are showing the effects just with continuum forces. Then we understand here what is difference between gravimeters and pendulums for showing mysterious effect of 
the pendulums.

Even the solar wind mystery is strongly on this procedure and electric charge asymmetry will result directly the solar wind and even the solar wind acceleration. Parker (1958) theorized that since gravity weakens as distance from the Sun increases, the outer coronal atmosphere escapes supersonically into interstellar space. But it is clear that early models of the solar wind relied primarily on thermal energy to accelerate the material, alone cannot account for the high speed of solar wind. An additional unknown acceleration mechanism is required. In the late 1990s the Ultraviolet Coronal Spectrometer (UVCS) instrument on board the SOHO spacecraft observed the acceleration region of the fast solar wind emanating from the poles of the Sun and found that the wind accelerates much faster than can be accounted for by thermodynamic expansion alone. Parker's model predicted that the wind should make the transition to supersonic flow at an altitude of about 4 solar radii from the photosphere (surface); but the transition (or "sonic point") now appears to be much lower, perhaps only 1 solar radius above the photosphere, suggesting that some additional mechanism accelerates the solar wind away from the Sun. Then the acceleration of the solar wind is still not understood and cannot be fully explained by Parker's theory.

$\mathrm{AC}$ voltage application is not alone way to exclude excess electrons from links with protons suppose the heat is too a next way. The heat is reason to increase the entropy in the matter and cut the electron-proton links. Then after passing from a transition point and the bound of entropy, the excess electrons will be separated from atomic level links because that in high temperature, the atomic links tends to be in number equilibrium resulting equality of the electrons and protons numbers as a critical point to appear observable high temperature plasma's electrical features.

Then Plasma is in reality a normal matter included to the equal number of the electrons and protons and how much the temperature is higher, the number equality of the electrons with protons is nearer to perfect and expanding of the universe by electric charge asymmetry is itself a reason for the reality that our universe is mainly a plasma and expansion of the universe is appeared by plasma-plasma antigravity almost three times as great as the gravity.

When we consider the Sun as a hot plasma below the convection zone then it should be an electric acceleration from the Sun to each normal 
plasma out of the Sun where the unlinked excess electrons are shielded by electric double layer of the Sun surface. Clearly the shielding effect of the Sun's surface double layer is in the scale of the solar system and the Sun's electric double layer is reason to appear antigravity between the Sun and a normal plasma in the inertia system of the solar system. We should notice that the Sun's surface double layer is working at the solar system scale to shield unlinked excess electrons and then the electric force between the Sun and a next star in the inner of the Milky way galaxy is pure gravity.

The Sun's core and radiation zone are almost $98 \%$ of Sun's mass and then ever exist a repulsive electric force by the Sun on the normal plasma out of the Sun surface. From Nipher experiments and calculation of Hubble law by electro antigravity it was resulted that the repulsive force of number neutral body is almost 3 times as great as the pure gravity and then additional force of gravity and antigravity results an acceleration on a normal test body equal to:

$a_{r}=-2 \frac{G M}{r^{2}}$.

Now we can calculate the speed of plasma by Sun force ranging from Sun's surface (radius $R$ ) to a distance $r$ so that:

$v^{2}=2 \int_{R}^{r} a_{r} \mathrm{~d} r$.

And by substituting Eq. (21) in the Eq. (22) we can calculate the speed of solar wind as (Fig. 4):

$v^{2}=-4 G M\left(\frac{1}{R}-\frac{1}{r}\right)$.

This is remembering the Parker diagrams of the solar wind speed (Parker, 1965, 2001) and the Sun's magnetic field is reason to appear spiral motion of the solar wind at the solar system. It is so excellent that the same force which generating the universe expansion is generating exactly solar wind at the same amplitude and this is a very strong confirmation for fundamental charge asymmetry.

This phenomenon may be used to build the plasma flying saucers or generally normal body flying saucers because we can use some devices included 


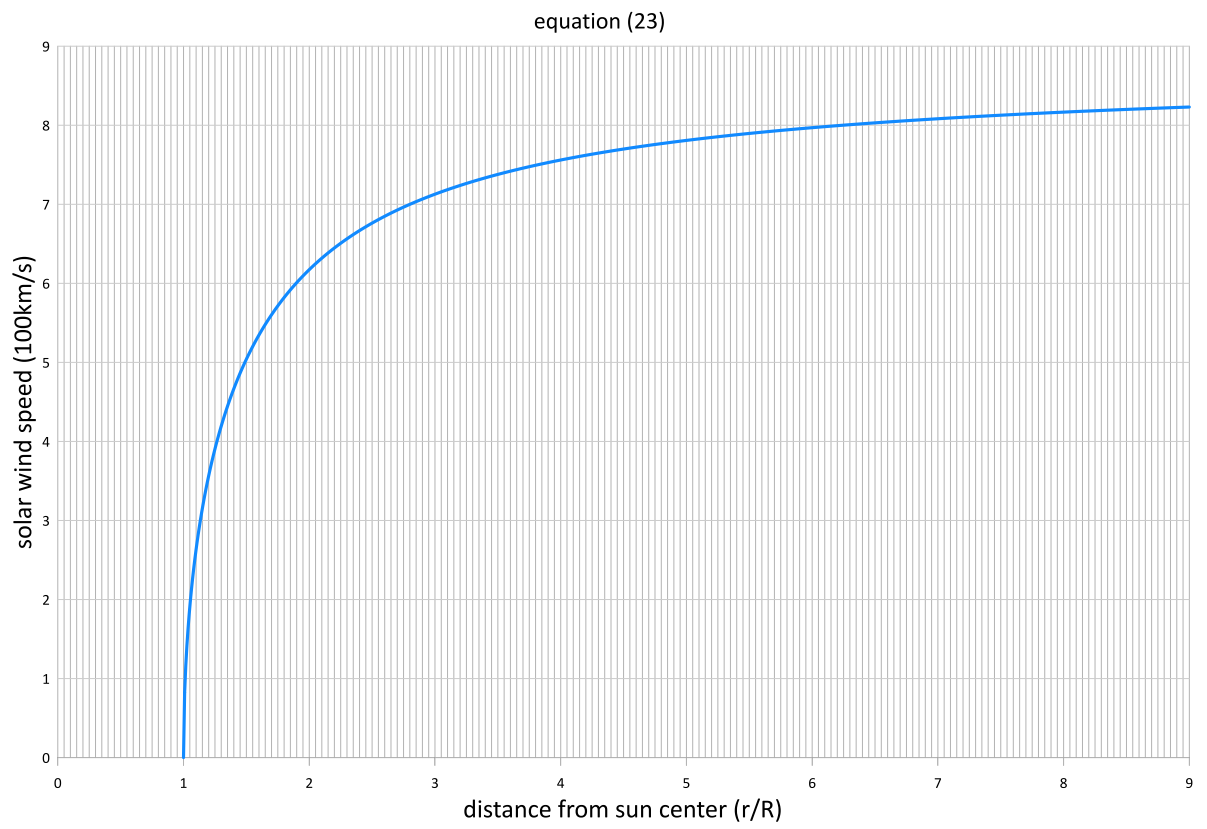

Fig. 4. Speed of plasma in the absence of the Sun's atmospheric dielectric.

to the normal body under the Sun electro anti-gravity similar to the solar wind. Then we will have very rapid interplanetary plasma flying saucers. Continuation of this especial issue will connect to the capacitors mysteries effect and also quantization and boundary dependency of the forces which we can continue it in the book "MOED": Modification of electro dynamics with Mach inertia principle and electro anti-gravity (Lutephy, 2016). There are important questions we have answered there for that if we want to continue here, the paper will inter to some new sections out of our target here. Of course there are other phenomena which we have not explained them here for example Sun flairs and Sun spots and coronal mass rejection (CMR) which for shortening the paper we have explained them in the book "MOED": Modification of electro dynamics with Mach inertia principle and electro anti-gravity.

Of course we should notice that the completeness of the normal body $\left(N_{e}=N_{p}\right)$ is dependent to the temperature of the plasma means how much the temperature to be rather, the plasma is nearer to a perfect equality for 
number of the electrons and protons. Also at the coronal radii, the repulsive force on the plasma becomes rapidly decreased reasoned by the Sun's atmospheric dielectric and this is resulting the corona as an equilibrium between the gravity and antigravity at the corona.

Too the solar wind is observed to exist in two states, termed the slow solar wind and the fast solar wind, though their differences extend well beyond their speeds. In near-Earth space, the slow solar wind is observed to have a velocity of $300-500 \mathrm{~km} / \mathrm{s}$, a temperature of $1.4-1.6 \times 10^{6} \mathrm{~K}$ and a composition that is a close match to the corona. By contrast, the fast solar wind has a typical velocity of $750 \mathrm{~km} / \mathrm{s}$, a temperature of $8 \times 10^{5} \mathrm{~K}$ and it nearly matches the composition of the Sun's photosphere (Geiss, 1995). As we see here, the high temperature solar wind is for slow solar wind in contrast with some theoretical alternatives for corona mystery.

On the other hand, the Sun has an atmosphere extended between surface and a radii $r_{t}$ in the top of the chromosphere mainly included to the plasma and then we have atmospheric dielectric effect generated by Sun's atmosphere. Of course we should notice that the atmosphere of the Sun can't be a dielectric for Sun's inertial electric charge in usual case. But the Sun inertial electric charge is very big at surface position on the plasma (Earth mantle too is a dielectric for inertial charge because of its very width and dense structure). Such a very big amplitude of the electric force is enable to create a noticeable polarization at the Sun atmosphere and then resulting dielectric effect on the Sun's inertial electric force. Then atmosphere of the Sun causes to decrease the Sun total inertial charge to a value as effective inertial charge of the Sun. It is clear that the width of the atmosphere is reason to increase the dielectric effect (Kamrin and Koval, 2012) and then the Sun effective inertial charge is decreased by distance from the Sun surface until the radii $r_{t}$, above the Sun atmosphere.

In this radii $r_{t}$, the atmospheric dielectric effect arrives to its maximum size and there is a chirality in this radii for effective inertial electric charge of the Sun so that by increase of the distance above this chirality radii, the effective inertial electric charge begins to increase again asymptotically arriving to the Sun total inertial charge. Then we have a chirality radii $r_{t}$ above the Sun atmosphere with minimum of Sun's effective inertial charge $q_{t}^{\&}$ there, because of maximum dielectric effect in this radii. Then above the chirality radii $r_{t}$ we have suddenly increase of the Sun effective electric 
charge asymptotically and below this transition radius we have exponentially increase of the Sun effective electric charge to a size equal to the Sun's total inertial charge at the Sun's surface. Then it is resulted that the asymptotic inertial electric charge of the Sun is equal with inertial electric charge below the atmosphere on the Sun's surface so that:

$q_{\text {sur }}^{\&}=q_{a s y}^{k}$.

We need a function for effective charge of the Sun that:

1. Sun inertial electric charge on the surface to be equal with Sun total inertial charge.

2. Sun asymptotic inertial electric charge to be equal with Sun total inertial charge.

3. The Sun effective inertial charge to be minimum $q_{t}^{\&}$ at the chirality radii $r_{t}$.

4. Exponentially increase of the inertial charge below the chirality radii until surface.

5. Rapid increase of the Sun's inertial charge above chirality radii asymptotically.

What we should know here it is the variation of the electric permittivity by distance from the Sun surface which it causes to vary the Sun effective inertial charge by distance.

At the atmosphere of the Sun we have a plasma dielectric feature enable to decrease highly the Sun's inertial electric force similar to the simple electric screening effect and also for plasma dielectric generated from a fluid medium we can use from the solution of Poisson equation (Kamrin and Koval, 2012) in the fluid that it results an exponential function as screened Poisson equation that:

$\varphi(r)=\frac{q}{4 \pi \varepsilon_{0} r} e^{-k_{0} r}$,

so that $\varphi$ here is electric potential energy of the number charge in a large scale sphere and $k_{0}$ is assumed constant for cosmic plasma and in reality this function is the same Yukawa potential in the strong nuclear force.

A simple analysis shows that all functions with mentioned characters above, are near to the diagram generated by below function that: 
$q_{\text {sun }}^{\&}=q_{t}^{\&}+\frac{4^{2}}{\pi^{2}}\left(q_{\text {sur }}^{\&}-q_{t}^{\&}\right) \arctan ^{2}\left(1-\frac{r_{t}^{n}}{r^{n}}\right)$.

It is manifest that $q_{t}^{\&}$ is minimum inertial electric charge of the Sun at chirality radii because that the minimum inertial electric charge is occurred at this equation where the function arc tan is zero and clearly asymptotic inertial electric charge is equal to the Sun total inertial electric charge.

In the Eq. (26), the Sun's surface inertial charge should be equal to asymptotic inertial electric charge and the Eq. (26) is in agreement with exponential increase, below the chirality radii until to the radius $R$ and too in agreement with rapid increase of the inertial electric charge above the chirality radii.

Geometrically the Eq. (26) is good approximate formula to show the Sun's effective inertial charge, above the Sun surface limited to the solar system and best fitting is showing that assumed power $n$ in Eq. (26) is suitable to be considered at $n=8$. Observationally it is good approximation to consider Sun's chirality radii $r_{t}$ in proportion to the Sun's radii $R$ as:

$r_{t} / R=1.08$.

Substituting Eqs. (24) and (27) in the Eq. (26) and $n=8$ and using the Sun's total inertial charge we result that

$q_{\text {sur }}^{\&}=5.92 q_{t}^{\&}$.

Then the Eq. $(26)$ in unit $\left(q_{t}, r_{t}\right)$ is

$q_{\text {sun }}^{\&}=1+8 \arctan ^{2}\left(1-\frac{1}{r^{8}}\right)$.

And this Eq. (29) geometrically in units $\left(r_{t}, q_{t}\right)$ is showing at Fig. 5.

By Eq. (8) we result for additional acceleration from the Sun on the plasma that:

$a_{r}=\frac{G M}{r^{2}}-\frac{q_{s u n}^{\&}}{r^{2}} \sqrt{3 G k}$.

Mixing the atmosphere dielectric effect at Eq. (29) with this simple formula implies an approximately suitable function for Sun's force on the plasma acceleration that: 


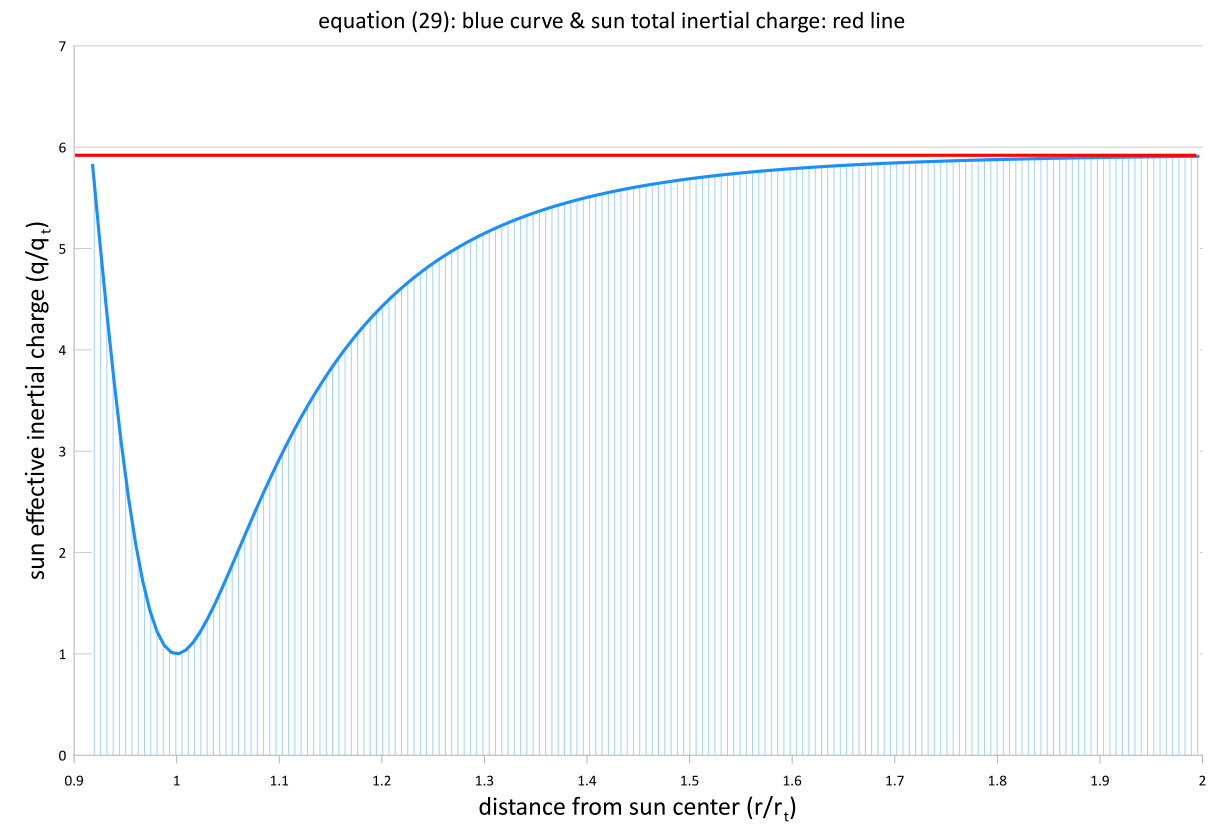

Fig. 5. Function of Sun effective inertial charge versus distance. The red line is the Sun's inertial charge in the absence of the dielectric effect of the Sun's atmosphere $(R=$ $\left.0.927 r_{t}\right)$.

$a_{r}=\left(\frac{G M}{r_{t}^{2}}\right)\left(\frac{r_{t}}{r}\right)^{2}\left[\frac{1}{2}-4 \arctan ^{2}\left(1-\left(\frac{r_{t}}{r}\right)^{8}\right)\right]$.

This function is visible at Fig. 6 .

Then we see that how much the beginning point of the solar wind is nearer to the Sun's surface, the speed of the solar wind will be asymptotically greater, as a strong verification for acceleration of the plasma by Sun's electric force. When the plasma is speeding from the coronal shell it is manifest that acceleration of the plasma is lower and proportionally the asymptotic speed is lesser. Then it should be appeared two level of the solar winds here so that one at the photosphere below the corona and next the plasma accelerating from the outer regions of the corona. Reason of this separation is that at coronal layer, the equilibrium between gravity and antigravity is zero and then the Sun has no electric force on the plasma at the corona center and this effect divides the solar wind to the fast and slow 


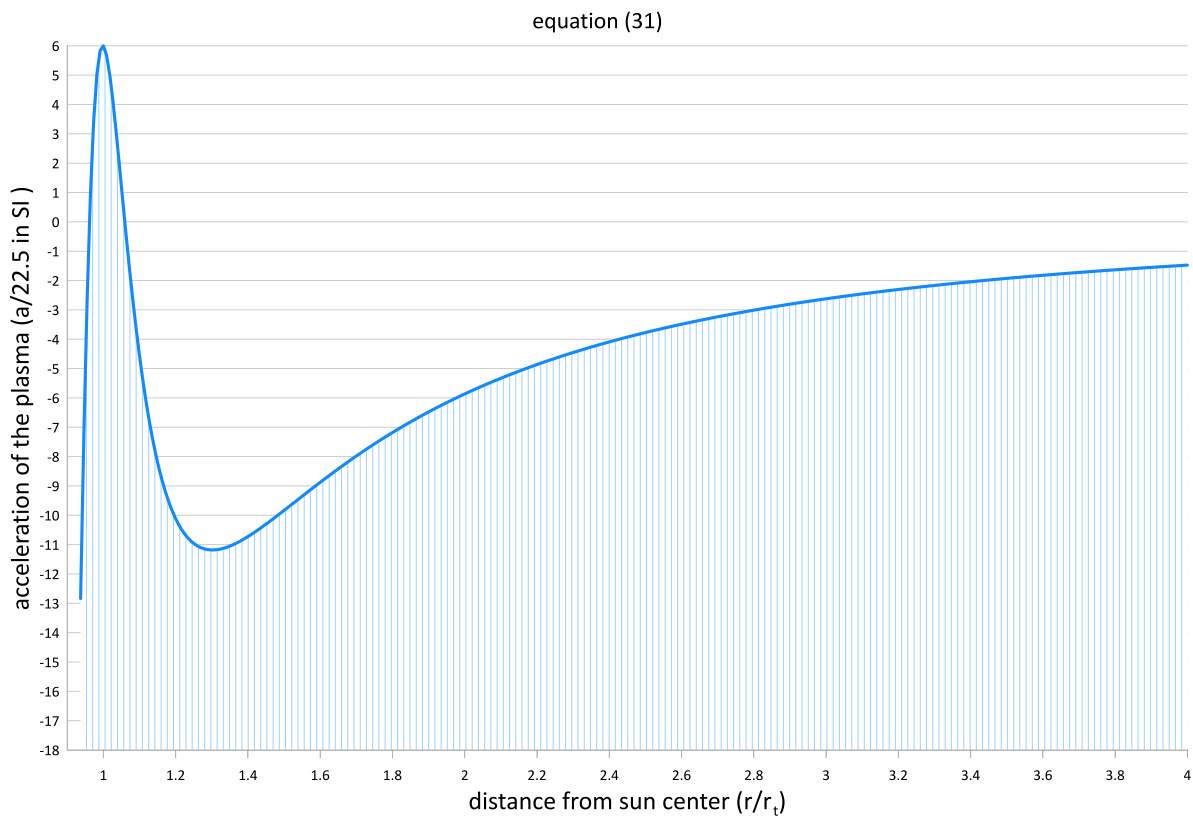

Fig. 6. Diagram of the Sun effective acceleration on the plasma in unit $r_{t}$ and acceleration unit $22.5 \mathrm{~m} / \mathrm{s}^{2}$.

levels completely compatible with the observations and records for solar wind.

On the other hand, for acceleration by Sun's inertial electric force on a normal plasma we have:

$a_{r}=\left\{\begin{array}{l}+\mid r_{c}<r<r_{t} \\ 0 \mid r_{c} \\ -\mid r\left(-a\left(r_{t}\right)\right)<r<r_{c}\end{array}\right.$.

So that $r_{c}$ is corona radius. Then plasma is pressed in a shell between maximum gravity and a radius with inverse size at antigravity which in the middle of this shell, the acceleration is zero as equilibrium point between gravity and antigravity.

We should notice that gravity is almost maximum at the chirality radius $r_{t}$ because that at this radius we have minimum effective inertial charge. 
Observations and mathematical calculations are showing that it is good approximation $d=R / 10$ so that $d$ is width of the assumed corona shell and for hydrostatic equilibrium it needs the pressure of the force on the shell to be equal with pressure of the coronal plasma inside the shell. According to the state equation $p=\rho k T / \mu$ we have:

$\frac{\bar{m} a\left(r_{t}\right)}{4 \pi r_{c}^{2}}=\frac{m k T}{4 \pi r_{c}^{2}\left|r\left(-a\left(r_{t}\right)\right)-r_{t}\right| \mu}$.

So that $\bar{m}$ is average mass of the corona shell affected in each moment by force in assumed shell between maximum gravity and inverse antigravity and $m$ is total mass inside the shell and then:

$T=\frac{\mu}{k} \frac{\bar{m}}{m}=\left|r\left(-a\left(r_{c h}\right)\right)-r_{t}\right| a\left(r_{c h}\right)$.

In agreement with equation of the state we have $k=8300$ and $\mu=1.5$. In the maximum effect, the average size of the mass affected in each moment by force is equal with total mass and then:

$T=2 \times 10^{6} \mathrm{~K}$.

And this is in agreement with observations as high temperature of the Sun's corona as a very strong verification for the reality that the corona is where the gravity and antigravity are in equilibrium in amplitudes and as a very clear verification, again for fundamental charge asymmetry (Fig. 7).

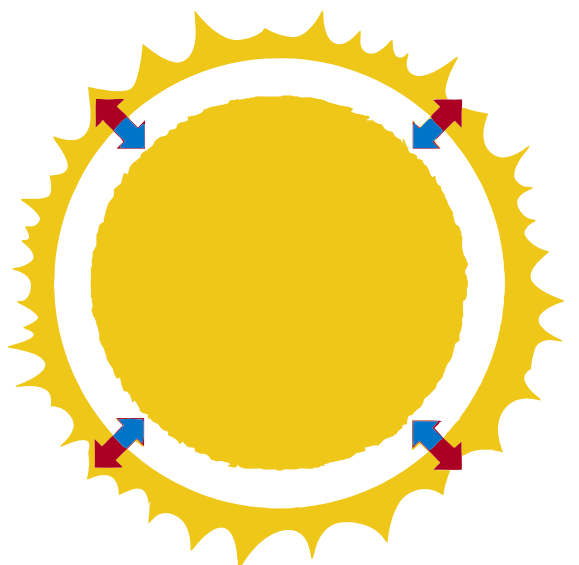

Fig. 7. Corona as equilibrium between gravity and antigravity. 
Referring to the Parker diagrams of the solar wind shows a maximum amplitude of the asymptotic speed at $1000 \mathrm{~m} / \mathrm{s}$ for solar wind at very high temperature whereas the calculation by electric charge asymmetry on the base of the Nipher experiments and modified in size by expanding universe is showing a maximum amplitude at $800 \mathrm{~m} / \mathrm{s}$ calculated by Eq. (23). Then the calculated electric charge asymmetry by Hubble constant is showing that the normal matter in the universe is not perfect suppose universe matter should be $66 \%$ of a complete high temperature plasma. Then by electrically neutralization of the normal matter of the universe along the time and then decrease of the normal matter of the universe, ultimately the expansion of the universe will end and it will be started the contraction of the universe when the antigravity is diminished. Contraction of the universe will generate very condensed high temperature universe in a very small volume. Then the normalization of the matter will be increased again reasonable to start again the expansion of the universe consequently. This section is discussed in detail at the book "MOED" (Lutephy, 2016) and such a periodical acceleration of the universe is resolving too the paradox of the singularity in early universe.

The plasma tail of the comets is too under the effect of the Sun's electric force and then the comets plasma tail is directed to out radially. Biermann postulated that comets tail out ward direction happens because the Sun emits a steady of particles that pushes the comet's tail away (Biermann, 1951). Of course it seems that Paul Ahnert was the first to relate solar wind to comets tail direction based on the observations of the comet WhippleFedke. By the way, these theories are weak in comparison with the reality of electric repulsive force from the Sun on the plasma tail of the comets and reason is that the contact of the solar wind to comet's tails will ride away all material tails whereas here just the plasma tail is directed away.

Ultimately with return to the magnetic field of spinning bodies, the used body should be normal until to result inertial magnetic field and in the terrestrial planets, naturally we have a normal rotating shell as the outer core and this shell will generate inertial magnetic field manifestly.

For a neutron star which it be completely normal, calculations by Eq. (15) we obtain at the poles that:

$B=70 \frac{R^{2}}{T}$. 
So that $R$ is radius of neutron star and $T$ is its period. This is completely in agreement with observations of the magnetic fields for magnetars which is a type of neutron star believed to have extremely powerful magnetic field $\left(10^{10}-10^{11} \mathrm{~T}\right)$.

This is showing that the magnetars are completely normal neutron stars and short age of the magnetars verifies this reality for that a completely normal matter will be neutralized electrically rapidly and manifestly different percentages of the normalization in the neutron stars will generate wide range of the magnetic field for neutron stars completely in agreement with observations of the magnetic fields for neutron stars as visible above that the binary neutron star PSR1913+16 is just $2 \%$ that of a complete normal body.

But the planets magnetic fields are relevant to their conductive outer cores in terrestrial planets and the conductive cores of the Jovian planets, whether theoretical metallic hydrogen or probable metallic inner regions verified in the paper (Lutephy, 2018) reasonable for strong interplanetary electromagnetic inductions generating relevant geomagnetic jerks and LOD variations and gravity anomalies in the Earth position.

Inner cores of the planets are electrically neutral, reasoning to keep ever the outer cores as a normal shell. But inner core is tiny in comparison with outer core for all planets except probably at Mars and then as a good approximate formula for magnetic field of the terrestrial planets we can integrate over whole of the core whereas that inner core doesn't generate magnetic field. Then from Eq. (15) for magnetic field of the terrestrial planets we have:

$B=\frac{8 \pi}{3 c^{2}} \sqrt{3 G k} \frac{\omega}{R^{3}} \int_{\text {outer-core }} \rho r^{4} \mathrm{~d} r$.

From planets magnetic field observed amplitudes embedding to Eq. (37) we obtain the radius of the electrically conductive core of these planets as the answers visible at the Fig. 8.

We observe that the percentage of the outer core radiuses of the giant planets per their radiuses is equal for all giant planets as a very strong confirmation for stationary magnetic field of spinning conductive regions in the interior of the planets and the calculated radiuses for CMB at the terrestrial planets is completely in agreement with observations. Then all 


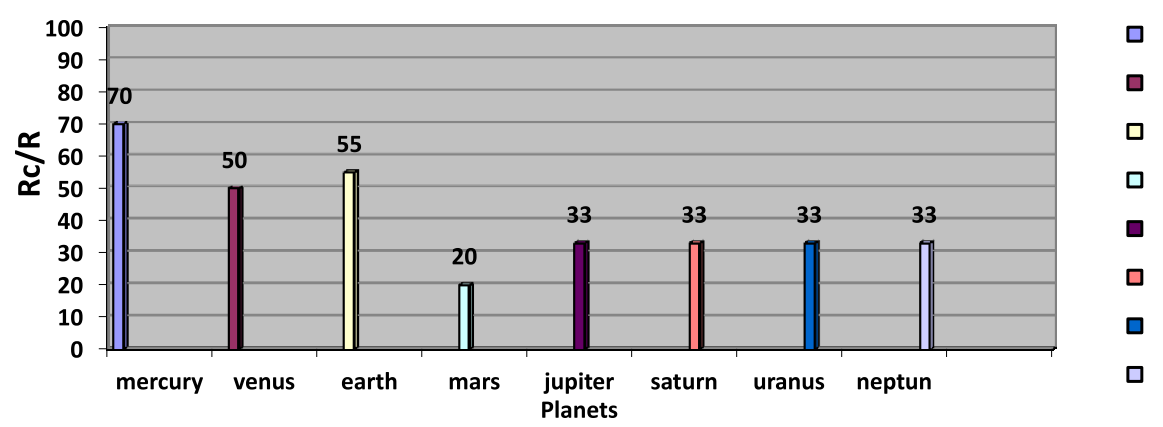

Fig. 8. Diagram of radius of conductive core of the planets per planets radius in $\%$ calculated by Eq. (37).

planets except Mars are in agreement with Eq. (37). As it was stated above, the Mars outer core may be transferred highly to the inner core. It is impossible that the radius of the outer core to be small because that the Mars has had a noticeable paleomagnetic field.

For Sun like stars means yellow dwarfs it was resulted above that outer convection zone is normal. Then for inertial magnetic field with assuming total convection zone as a normal shell we obtain that:

$B=\frac{8 \pi}{3 c^{2}} \sqrt{3 G k} \frac{\omega}{R^{3}} \int_{\text {convection }} \rho r^{4} \mathrm{~d} r$.

According to Dalsgaard density profile of the Sun (Christensen-Dalsgaard et al., 1996), approximately we have:

$\rho=10\left(5 \times 10^{4}\right)^{1-r / R} \mid r \leq 0.95 R$.

This density profile is deviated about the surface, but it is a good approximation and by embedding Eq. (39) into Eq. (38) we obtain the Sun's inertial magnetic field if total convection zone was normal from below formula:

$B=\frac{8 \pi k \omega}{3 c^{2} R^{3}} \sqrt{\frac{3 G}{k}} \int_{R_{c o n}}^{R}\left[10\left(5 \times 10^{4}\right)^{1-r / R}\right] r^{4} \mathrm{~d} r$.

So that $R_{c o n}$ is bottom radii of conductive zone and by this equation we obtain an answer for Sun's magnetic field near to the observations for Sun's 
magnetic field. But Sun's magnetic features especially its dipole reversal is showing that the self-consistent dynamo is working there and this reversal is not an historical report suppose it is doing cyclical in each eleven years presently. Then strongly dynamo is prevailing mechanism in the generation of the Sun's magnetic field and this is a real paradox here because that both of the mechanisms are in agreement whereas that it seems the dynamo is the main effect on the Sun's magnetic field.

We should notice that the convection zone is divided to the two shells which inner convection zone is electrically neutral low temperature plasma, not producing the magnetic field. Then stationary magnetic field will be generated just by rotation of the outer convection zone and this is a confirmation why in the Sun, the stationary magnetic field is weaker than that of what we measure it for total convection zone by Eq. (40) responsible to resolve the paradox.

On the other hand, we have used from Dalsgaard model (ChristensenDalsgaard et al., 1996)) to calculate stationary magnetic field of the Sun generated by rotating convection zone as a spinning normal body and Dalsgaard model is based all on the pure gravity whereas that outer convection zone is being antigravity two times as great as the gravity, yet not electrically neutralized by excess electrons. Then clearly we need to revise the Sun's density profile and rewriting the density dependent equations with assuming that the outer convection zone is antigravity as a reason to decrease the density of the matter in the convection zone means the density in the outer convection zone is ever lesser than what calculated by Dalsgaard model. Then real size of the density profile will result lesser amplitude for stationary magnetic field generated by outer convection zone for Sun. This is a next reason for reality that the magnetic field source of the Sun should be mainly self-consistent dynamo.

Of course we should notice that by increase of the temperature, the electric conductivity will decrease and decrease of electric conductivity is reason to appear condition for gradual electronic flows in the convection zone by self-consistent dynamo. Then the reversal mechanism probably has been doing in the terrestrial planets when the outer core has been hotter and then self-consistent dynamo in the planets have been reduced before and prevailing mechanism is now rotation of the normal interior regions of the planets. But about the Sun, we see a current reversal and then strongly the 
self-consistent dynamo is prevailing mechanism in the Sun in proportion to the normal body spinning magnetic field. In reality ever there are a complementary mechanism of the self-consistent dynamo and spinning normal body, aligned each other to generate celestial bodies magnetic field. Sometimes dynamo is prevailing and some times normal body spinning.

Referring to the above results and in detail to the book "MOED" (Lutephy, 2016), the universe expansion rate is dependent on the universal distribution of the number charges (universal electric potential energy) and percentage of the universe normal matter (equal number of electrons and protons) and also the density of universe and ultimately it is deduced that the universe expansion is not accelerating. The discovery of accelerating expansion led to the widespread acceptance of the idea that our Universe is dominated by a mysterious force called dark energy. But now physicists are questioning the conclusion, and they have a much larger dataset to back them up. Now, a team of scientists led by Professor Subir Sarkar of Oxford University's Department of Physics has cast doubt on this standard cosmological concept. Making use of a vastly increased data set - a catalogue of 740 Type Ia supernovae, more than ten times the original sample size - the researchers have found that the evidence for acceleration may be flimsier than previously thought, with the data being consistent with a constant rate of expansion. The study is published in the Nature Journal Scientific Reports by J. T. Nielsen, A. Guffanti and S. Sarkar (Nielsen et al., 2016).

In the book "MOED" (Lutephy, 2016) we have a pure argument for electric charge asymmetry at the Earth position correlated to the universal electric potential energy obtained by largest material concentration in the universe for Earth which it is local supercluster "the Laniakea" discovered newly. The Laniakea Supercluster is the galaxy supercluster that is home to the Milky Way and approximately 100,000 other nearby galaxies. it was defined in September 2014, when a group of astronomers including R. Brent Tully of the University of Hawaii, Hélène Courtois of the University of Lyon, Yehuda Hoffman of the Hebrew University of Jerusalem, and Daniel Pomarède of CEA Université Paris-Saclay published a new way of defining superclusters according to the relative velocities of galaxies. The Laniakea Supercluster is largest concentrated local structure at the observable universe so that such a concentrated matter can generate noticeable scalar electric potential to generate electric charge asymmetry at the Earth 
position emerged by the electric binding energy of the quarks at the proton (Lutephy, 2016) arriving to the below formula that:

$q_{e-p}^{\&}=e \frac{\sqrt{M_{p} / R_{p}}}{\sqrt{\sqrt{2 M_{\text {Laniakea }}}}} \cong 2.5 \times 10^{-37} \mathrm{C}$.

So that $M_{p}$ is the proton mass and $R_{p}$ is the proton radius and $e$ is fundamental electric charge.

In reality the electric charge asymmetry is not at the same value everywhere in the cosmos suppose it may be different in different positions and also the electric charge asymmetry is not ever constant in time suppose at different times maybe different in size, reasonable for inflation theory (Guth, 1997) discussed in detail at the book "MOED" (Lutephy, 2016).

Inflation is a theory of exponential expansion of the space in early universe. Inflation was a hypothetical very brief episode of highly accelerated expansion enable to explain some features of the universe as noted by Alan Guth himself that:

"I will begin by giving a quick rundown of how inflation works... The key idea - the underlying physics - that makes inflation possible is the fact that most modern particle theories predict that there should exist a state of matter that turns gravity on its head, creating a gravitational repulsion. This state can only be reached at energies well beyond those that we can probe experimentally, but the theoretical arguments for the existence of the state are rather persuasive. It is not merely the prediction of some specific theory, but it is the generic prediction for a wide class of plausible theories. Thus, gravity does not always have to be attractive. The gravitational repulsion caused by this peculiar kind of material is the secret behind inflation. Inflation is the proposition that the early universe contained at least a small patch that was filled with this peculiar repulsive-gravity material. There are a variety of theories about how this might have happened, based on ideas ranging from chaotic initial conditions to the creation of the universe as a quantum tunneling event... Once the patch exists it starts to rapidly expand because of its internal gravitational repulsion."

And in relation with Alan Guth inflation theory (Guth, 1997) we see here that the matter is possible to be transferred between the gravity and antigravity in different conditions appropriate for inflation theory. Manifestly 
early universe has been different highly and then the charge asymmetry in early universe has been very different in size and in reality we obtain that the charge asymmetry is much bigger in early universe in agreement with inflation theory.

\section{Conclusion}

Experimental results in laboratories and phenomenological results in the solar system and the cosmos, are showing the evidences of the fundamental electric charge asymmetry as the origin of a section of the planets and stars magnetic field accompanied with self-consistent dynamo. Also we see that the electric charge asymmetry as a difference between electron and proton electric charges is responsible for many number of the planetary and cosmological big unsolved puzzles. For example, Cosmic microwave background anisotropy and expanding universe and accelerating expansion of the universe and solar wind and solar wind acceleration and Sun's corona and missing neutrinos and inflation theory and singularity paradox and comets plasma tail direction and pendulums mysterious precessions. Too we have suitable interpretations for some mysterious experimental results for example Nipher experiments electro antigravity and Eugene Podkletnov superconducting disk antigravity and also we have proposed some experiments to do in the laboratories.

Acknowledgements. We offer this article to the spirits of the scientists, Francis E. Nipher, Maurice Allais, Patrick Blackett, Eugene N. Parker, Erwin Saxl, Thomas Townsend Brown, Arthur Schuster, Herman Bondi and other relevant scientists who have developed the science by their hard trying.

\section{References}

Allais M., 1959a: Should the Laws of Gravitation Be reconsidered? Part I - Abnormalities in the Motion of a Paraconical Pendulum on an Anisotropic Support. Aero/Space Engineering, 46-52.

Allais M., 1959b: Should the Laws of Gravitation Be reconsidered? Part II - Experiments in Connection with the Abnormalities Noted in the Motion of the Paraconical Pendulum With an Anisotropic Support. Aero/Space Engineering, 51-55. 
Allais M., 1999: The Allais effect and my experiments with the paraconical pendulum (1954-1960). Memoir C-6083 prepared for NASA (report).

Andrault D., Monteux J., Le Bars M., Samuel H., 2016: The deep Earth may not be cooling down. Earth and planetary science letters, 443, 195-203.

Assis A. K. T., Silva H. T., 2000: Comparison between Weber's electrodynamics and classical electrodynamics. Pramana, 55, 3, 393-404.

Bahcall J. N., 1964: Solar Neutrinos I. Theoretical. Phys. Rev. Lett. 12, 300-302.

Biermann L., 1951: Kometenschweife und solare Korpuskularstrahlung. Zeitschrift für Astrophysik, 29, 274 (in German).

Blackett P. M. S., 1947: The magnetic field of massive rotating bodies. Nature 159, $658-666$.

Blackett P. M. S., 1952: A negative experiment relating to magnetism and the Earth's rotation. Phil. Trans. R. Soc., A 245, 309-370.

Christensen-Dalsgaard J., Däppen W., Ajukov S. V., Anderson E. R., Antia H. M., Basu S., Baturin V. A., Berthomieu G., Chaboyer B., Chitre S. M., Cox A. N., Demarque P., Donatowicz J., Dziembowski W. A., Gabriel M., Gough D. O., Guenther D. B., Guzik J. A., Harvey J. W., Hill F., Houdek G., Iglesias C. A., Kosovichev A. G., Leibacher J. W., Morel P., Proffitt C. R., Provost J., Reiter J., Rhodes E. J. Jr., Rogers F. J., Roxburgh I. W., Thompson M. J., Ulrich R. K., 1996: The current state of solar modeling. Science, 272, 5266, 1286-1292, doi: 10.1126/science.272.5266 .1286.

Cowling T. G., 1934: The Magnetic Field of Sunspots. Monthly Notices of the Royal Astronomical Society, 94, 39-48, doi: 10.1093/mnras/94.1.39.

Davis R. Jr., 1964: Solar Neutrinos. II. Experimental. Phys. Rev. Lett., 12, 303-305.

Geiss J., Gloeckler G., Von Steiger R., 1995: Origin of the solar wind from composition data. Space Science Reviews, 72, 1-2, 49-60, doi: 10.1007/BF00768753.

Gilman P. A., 2000: Fluid Dynamics and MHD of the Solar Convection Zone and Tachocline: Current Understanding and Unsolved Problems (Invited Review). Helioseismic Diagnostics of Solar Convection and Activity, p. 27.

Guth A. H., 1997: The inflationary-universe. Reading, Massachusetts, Perseus books, ISBN 0-201-14942-7.

Hillas A. M., Cranshaw T. E., 1959: A Comparison of the Charges of the Electron, Proton and Neutron. Nature, 184, 892-893, doi: 10.1038/184892a0.

Jeverdan G. T., Rusu G. I., Antonescu V. I., 1961: Preliminary data about the behavior of a Foucault pendulum during the Sun eclipse of 15 February 15, 1961 (Date preliminare asupra comportarii unui pendul Foucault in timpul eclipsei de soare de la 15 februarie 1961). Annals of the Alexandru Ioan Cuza University, 7, 2, 457 (in Romanian).

Jeverdan G. T., Rusu, G. I., Antonescu V. I., 1981: Experiments using the Foucault pendulum during the solar eclipse of 15 February, 1961 (PDF). The Biblical Astronomer, 1, 55, 18-20.

Kamrin K., Koval G., 2012: Nonlocal Constitutive Relation for Steady Granular Flow. Physical Review Letters, 108, 17, 178301, doi : 10.1103/PhysRevLett.108.178301. 
Lutephy M., 2016: MOED: Modification of electric dynamics by Mach's inertia principle and electro anti-gravity. Edition 1st. ISBN: 9781537165691.

Lutephy M, 2018: Interplanetary external driven quasidynamo as the origin of geomagnetic jerks correlated with length of day and gravity anomaly. Contributions to Geophysics and Geodesy, 48, 1, 23-74.

Lyttleton R. A., Bondi H., 1959: Physical consequences of a general excess of charge, Proc. Roy. Soc. Lond., A252, 1270, 313-333, doi: 10.1098/rspa.1959.0155.

Mach E., 1872: Die Geschichte und Wurzel des Satzes von der Erhaltung der Arbeit. Prague (in German).

Mach E., 1911: History and Root of the Principle of the Conservation of Energy. Open Court, Chicago.

Mach E., 1883: Die Mechanik in ihrer Entwicklung Historisch-Kritisch Dargestellt (in German).

Mach E., 1960: The Science of Mechanics. Open Court.

Michelson A. A., Morely E. W., 1887: On the relative motion of the Earth and the luminiferous Eather. American Journal of Science, 34, 333-345.

Mishra D. C., Vyaghreswara Rao M. B. S., 1997: Temporal variation in gravity field during solar eclipse on 24 October 1995. Current Science, 72, 11, 782-783.

Moffatt H. K., 1978: Magnetic Field Generation in Elecrically Conducting Fluids, Cambridge University Press.

Nielsen J. T., Guffanti A., Sarkar S., 2016: Marginal evidence for cosmic acceleration from Type Ia supernovae. Scientific Reports, 6, 35596.

Nipher F. E., 1916: Gravitation and electrical action. Trans. Acad. Sci. St. Louis, 163175 .

Nipher F. E., 1917: Gravitational Repulsion. Trans. Acad. Sci. St. Louis, XXIII, 5.

Nipher F. E., 1918: Can Electricity Destroy Gravitation. Electric Experimenter.

Nipher F. E., 1920: New evidence of a relation between gravitation and electrical action and of local charges in the electrical potential of the Earth. Trans. Acad. Sci. St. Louis, XXVII, 383-387.

Parker E. N., 1958: Dynamics of the Interplanetary Gas and Magnetic Fields. The Astrophysical Journal, 128, 1, 664, doi: 10.1086/146579.

Parker E. N., 1965: The dynamical theory of the solar wind. Space Science Reviews, 4, 666-708.

Parker E. N., 2001: A history of the solar wind concept. The Century of Space Science, 225-255, Kluwer Academic Publishers.

Pippard A. B., 1988: The parametrically maintained Foucault pendulum and its perturbations. Proc. R. Soc. Lond., A 420, 81-91.

Podkletnov E., Nieminen R., 1992: A possibility of gravitational force shielding by bulk YBa2Cu3O7-x superconductor. Physica C, 203, 3-4, 441-444.

Podkletnov E., Vuorinen P. T., 1996: Gravitation shielding properties of composite bulk YBa2Cu3O7-x superconductor below $70 \mathrm{~K}$ under electromatic field. Journal of Applied Physics D. Retrieved April 29, 2014. 
Podkletnov E., 1997: Weak gravitation shielding properties of composite bulk YBa2Cu3O7-x superconductor below $70 \mathrm{~K}$ under e.m. field. Retrieved April 29, 2014. This is believed to be substantially the same paper accepted for publication in 1996 by Journal of Physics D which was later withdrawn by the author.

Popescu V. A., Olenici D., 2007: A confirmation of the Allais and Jeverdan-Rusu-Antonescu effects during the solar eclipse from 22 September 2006, and the quantization of behaviour of pendulum. 7th Biennial European SSE Meeting. Røros, Norway, Society for Scientific Exploration.

Ryskin G., 2009: Secular variation of the Earth's magnetic field: induced by the ocean flow? New journal of physics, 11, 6, 063015, doi : 10.1088/1367-2630/11/6/063015.

Sachs M., Roy A. R., Eds., 2003: Mach's Principle and the Origin of Inertia. Apeiron.

Sanders R. H., 1996: The Published Extended Rotation Curves of Spiral Galaxies: Confrontation with Modified Dynamics. The Astrophysical Journal, 473, 1, 117-129.

Saxl E. J., 1964: An electrically charged torque pendulum. Nature, 203, 4941, 136-138, doi : $10.1038 / 203136 \mathrm{a} 0$.

Schuster A., 1912: A critical examination of the possible causes of terrestrial magnetism. Proc. Phys. Soc. Lond., 24, 121-137.

Sengupta S., Pal P. B., 1996: Constraints on cosmic charge asymmetry and neutrino charge from the microwave background. Phys. Lett., B365, 175-177, doi: 10.1016/ S0370-2693(96) 01532-8.

Sengupta S., 2000: Binary Pulsar PSR B1913+16 constraints the electron-proton charge asymmetry. Physics letters B., 484, 3-4, 275-277.

Tarduno J. A., Cottrell R. D., Watkeys M. K., Hofmann A., Dobrovine P. V., Mamajek E. E., Liu D., Sibeck D. G., Neukirch L. P., Usui Y., 2010: Geodynamo, Solar wind, and magnetopause 3.4 to 3.45 billion years ago. Science, 327, 5970, 1238-1240, doi: $10.1126 /$ science. 1183445.

Wang Q. S., Yang X. S., Wu C. Z., Guo G. H., Liu H. C., Hua C. C., 2000: Precise measurement of gravity variations during a total solar eclipse (PDF). Physical Review D, 62, 4, 041101. arXiv: 1003.4947, doi: 10.1103/PhysRevD.62.041101.

Wilson H. A., 1923: An experiment on the origin of the Earth's magnetic field. Proc. R. Soc. Lond., A 104, 451-455.

Zhou S. W., Huang B. J., Ren Z. M., 1995: The abnormal influence of the partial solar eclipse on December 24th, 1992, on the time comparisons between atomic clocks. Il Nuovo Cimento C, 18, 2, 223-236, doi: 10.1007/BF02512022. 